\title{
Numerical 3D constraints on convective eddy time-correlations: Consequences for stochastic excitation of solar $p$ modes
}

\author{
R. Samadi ${ }^{1,2}$, A. Nordlund ${ }^{3}$, R. F. Stein ${ }^{4}$, M. J. Goupil ${ }^{2}$, and I. Roxburgh ${ }^{1,2}$ \\ 1 Astronomy Unit, Queen Mary, University of London, London E14NS, UK \\ 2 Observatoire de Paris, LESIA, CNRS UMR 8109, 92195 Meudon, France \\ 3 Niels Bohr Institute for Astronomy, Physics, and Geophysics, Copenhagen, Denmark \\ ${ }^{4}$ Department of Physics and Astronomy, Michigan State University, Lansing, USA
}

Received 9 December 2002 / Accepted 30 March 2003

\begin{abstract}
A 3D simulation of the upper part of the solar convective zone is used to obtain information on the frequency component, $\chi_{k}$, of the correlation product of the turbulent velocity field. This component plays an important role in the stochastic excitation of acoustic oscillations. A time analysis of the solar simulation shows that a Gaussian function does not correctly reproduce the $v$-dependency of $\chi_{k}$ inferred from the 3D simuation in the frequency range where the acoustic energy injected into the solar $p$ modes is important $(v \simeq 2-4 \mathrm{mHz})$. The $v$-dependency of $\chi_{k}$ is fitted with different analytical functions which can then conveniently be used to compute the acoustic energy supply rate $P$ injected into the solar radial oscillations. With constraints from a 3D simulation, adjustment of free parameters to solar data is no longer necessary and is not performed here. The result is compared with solar seismic data. Computed values of $P$ obtained with the analytical function which fits best $\chi_{k}$ are found $\sim 2.7$ times larger than those obtained with the Gaussian model and reproduce better the solar seismic observations. This non-Gaussian description also leads to a Reynolds stress contribution of the same order as the one arising from the advection of the turbulent fluctuations of entropy by the turbulent motions. Some discrepancy between observed and computed $P$ values still exist at high frequency and possible causes for this discrepancy are discussed.
\end{abstract}

Key words. convection - turbulence - stars: oscillations - Sun: oscillations

\section{Introduction}

Solar oscillations are believed to be stochastically excited by turbulent convection in the outer part of the Sun. The excitation is caused by turbulent convective motions which generate acoustic energy which in turn is injected into the $p$ modes.

Models of stochastic excitation of stellar $p$ modes have been proposed by several authors (e.g. Goldreich \& Keeley 1977; Osaki 1990; Balmforth 1992; Goldreich et al. 1994). These models use simplified models to describe the dynamics of the turbulent medium. For instance these approaches (Goldreich \& Keeley 1977; Balmforth 1992) assume a Gaussian function for representing $\chi_{k}$, the frequency component of the correlation product of the turbulent velocity field. As pointed out by Samadi (2001), the way the component $\chi_{k}$ is modeled plays a crucial role in controlling the extent of the excitation region of a given mode and hence the total amount of acoustic energy injected into the mode. In the following, $\chi_{k}$ will also be referred to as the dymamic model of turbulence and $d y$ namic will refer to time-dependence or frequency-dependence.

Send offprint requests to: $\mathrm{R}$. Samadi, e-mail: reza.samadi@obspm.fr
Direct computations of the rate at which the solar $p$ modes are excited have been performed by Stein \& Nordlund (2001) using 3D simulations of the upper part of the solar convective zone. They found good agreement between their numerical results and the solar seismic observations. This direct but time consuming approach did not address the role of the dynamic properties of the turbulent medium on the excitation mechanism.

In contrast semi-analytical formulations for $P(v)$ offer the advantage of testing separately several properties entering the excitation mechanism. Here we consider the formulation by Samadi \& Goupil (2001, hereafter Paper I, see also Samadi 2001 for a summary) which includes a detailed treatment of the time averaged and dynamic properties of the turbulent convective medium.

The impact of the averaged properties have been investigated by Samadi et al. (2003). The authors used a 3D simulation of the upper part of the solar convective zone to constrain the averaged properties of the turbulent convective medium. The computed rates $P$ at which the solar $p$ modes are excited were found to be larger than those computed with a 1D mixinglength solar model but still underestimate the solar seismic data 
by a factor $\sim 2.5$. It was also found that the Reynolds tensor contributes about $20 \%$ of the total acoustic energy injected into the solar $p$ modes, in contrast with direct 3D estimations (Stein \& Nordlund 2001). These discrepancies were attributed to the assumed Gaussian function for the dynamic model of turbulence.

In the present paper we therefore derive an empirical dynamic model of turbulence obtained from a 3D simulation of the upper part of the solar convective zone and then study the consequences of using this model on the computed excitation rates $P$. We compare our computation with solar seismic data and finally obtain on an improved model of stochastic excitation.

The paper is organised as follows: The basic theoretical background and notations are recalled in Sect. 2. In Sect. 3, a 3D simulation of the upper part of the solar convective zone is used to characterise $\chi_{k}$ in the domain where stochastic excitation takes place. The inferred $v$-dependency of $\chi_{k}$ is compared with the Gaussian function and fitted with different nonGaussian functions. These functions are then used in Sect. 4 to compute the excitation rate $P$ for radial $p$ modes. The results are compared with solar seismic observations as provided by Chaplin et al. (1998) and with computations in which the Gaussian function is assumed. Section 5 is dedicated to discussions and conclusions.

\section{Stochastic excitation}

\subsection{The model of stochastic excitation}

We consider the model of stochastic excitation as described in Paper I and assume here - as in Samadi et al. (2003) - that injection of acoustic energy into the modes is isotropic and consider only radial $p$ modes. Accordingly, the rate at which a given mode with frequency $\omega_{0}$ is excited can be written as:

$$
\begin{gathered}
P\left(\omega_{0}\right)=\frac{\pi^{3}}{2 I} \int_{0}^{M} \quad \mathrm{~d} m \frac{\Phi}{3} \rho_{0} w^{4}\left\{\frac{16}{15} \frac{\Phi}{3}\left(\frac{\mathrm{d} \xi_{r}}{\mathrm{~d} r}\right)^{2} S_{R}\right. \\
\left.+\frac{4}{3}\left(\frac{\alpha_{s} \tilde{s}}{\rho_{0} w}\right)^{2} \frac{g_{r}}{\omega_{0}^{2}} S_{S}\right\}
\end{gathered}
$$

In Eq. (1), $\rho_{0}$ is the mean density, $\xi_{r}$ is the radial component of the fluid displacement adiabatic eigenfunction $\xi, I$ is the mode inertia (Eq. (19)), $\alpha_{s}=(\partial p / \partial s)_{\rho}$ where $p$ denotes the pressure and $s$ the entropy, $\tilde{s}$ is the rms value of the entropy fluctuations which are assumed to arise solely from turbulence, $g_{r}\left(\xi_{r}, r\right)$ is a function involving the first and the second derivatives of $\xi_{r}$ with respect to $r, \Phi$ is a mean anisotropy factor defined by Gough (1977) as

$\Phi(r) \equiv \frac{\overline{<\boldsymbol{u}^{2}>-<\boldsymbol{u}>^{2}}}{w^{2}(r)}$

where $\boldsymbol{u}$ is the velocity field, $<$. $>$ denotes horizontal average, $\overline{()}$ denotes time average, and $w(r)$ is the mean vertical velocity $\left(w^{2} \equiv \overline{<u_{z}^{2}>-<u_{z}>^{2}}\right)$. Expressions for $g_{r}\left(\xi_{r}, r\right)$ are given in Samadi et al. (2003).
The driving sources $S_{R}\left(r, \omega_{0}\right)$ and $S_{S}\left(r, \omega_{0}\right)$ arise from the Reynolds stress and the entropy fluctuations respectively:

$$
\begin{aligned}
S_{R}\left(r, \omega_{0}\right)= & \int_{0}^{\infty} \frac{\mathrm{d} k}{k^{2}} \frac{E(k, r)}{u_{0}^{2}} \frac{E(k, r)}{u_{0}^{2}} \chi_{k}\left(\omega_{0}, r\right) \\
S_{S}\left(r, \omega_{0}\right)= & \int_{0}^{\infty} \frac{\mathrm{d} k}{k^{2}} \frac{E(k, r)}{u_{0}^{2}} \frac{E_{s}(k, r)}{\tilde{s}^{2}} \\
& \times \int_{-\infty}^{+\infty} \mathrm{d} \omega \chi_{k}\left(\omega_{0}+\omega, r\right) \chi_{k}(\omega, r)
\end{aligned}
$$

where $u_{0}(r) \equiv \sqrt{\Phi / 3} w$ is introduced for convenience, $E(k, r)$ is the time averaged turbulent kinetic energy spectrum, $E_{s}(k, r)$ is the time averaged turbulent spectrum associated with the entropy fluctuations and $\chi_{k}(\omega, r)$ is the frequency-dependent part of the correlation product of the turbulent velocity field (see Sect. 2.2). In order to simplify the notation, we drop the explicit $r$ dependence of the quantities in Eqs. (1-4).

\subsection{The dynamic model of turbulence}

The dynamic model of turbulence is represented by $\chi_{k}(\omega)$. In order to give a precise meaning to $\chi_{k}(\omega)$, we recall first some theoretical relations. Excitation by Reynolds stresses involves $\phi_{i, j}(k, \omega)$, the Fourier transform of the second-order velocity correlations; here the indices $i$ and $j$ refer to any of the 3 directions of the velocity field. For incompressible, homogeneous and isotropic turbulence, $\phi_{i j}(k, \omega)$ has the form (Batchelor 1970):

$\phi_{i j}(k, \omega)=\frac{E(k, \omega)}{4 \pi k^{2}}\left(\delta_{i j}-\frac{k_{i} k_{j}}{k^{2}}\right)$

where $E(k, \omega)$ is the turbulent kinetic energy spectrum as a function of $k$ and $\omega$ and $\delta_{i j}$ is the Kronecker tensor. Following Stein (1967), $E(k, \omega)$ is decomposed as

$E(k, \omega)=E(k) \chi_{k}(\omega)$

where $\chi_{k}(\omega)$ satisfies the normalisation condition (Tennekes \& Lumley 1982, Chap. 8.1):

$\int_{-\infty}^{+\infty} \mathrm{d} \omega \chi_{k}(\omega)=1$

According to the decomposition of Eq. (6), $\chi_{k}(\omega)$ is - at fixed $k$ - the frequency component of $E(k, \omega)$.

According to Eqs. (5) and (6), $\chi_{k}(\omega)$ then represents the frequency dependence of $\phi_{i, j}(\boldsymbol{k}, \omega)$. In other words, $\chi_{k}(\omega)$ measures - in the frequency and $k$ wavenumber domains - the evolution of the velocity correlation between two distant points of the turbulent medium.

The same decomposition of Eq. (7) is assumed for $E_{s}(k, \omega)$. This leads to introducing $\chi_{k}^{s}$, the frequency-dependent part of the correlation product of the entropy fluctuation. For simplifying the computation of $P$, as in Paper I, we assume $\chi_{k}^{s}=\chi_{k}$. We have checked that $\chi_{k}^{s}$ and $\chi_{k}$ have almost the same behaviour in the region where excitation by entropy fluctuations is significant.

In the present work, we consider only the excitation of radial $p$ modes. Let $E_{z}(k, \omega)$ be the vertical component of the 
kinetic energy spectrum. We consider that $E_{z}(k, \omega)$ can be decomposed as $E(k, \omega)(\mathrm{Eq} .(7))$. For isotropic turbulence we then have $E(k, \omega)=3 E_{z}(k, \omega), E(k)=3 E_{z}(k)$ and $\chi_{k}^{z}=\chi_{k}$, which is equivalent to stating that the averaged and dynamic properties of the velocity field are the same in all 3 directions.

The anisotropy factor $\Phi$ - introduced in the expression for $P$, Eq. (1) - partially takes into account the spatial and temporal anisotropy of the turbulence $(\Phi=3$ corresponds to a isotropic turbulence). It has been found in Samadi et al. (2003) that $\Phi \simeq 2$ within the region where most of the excitation occurs. This shows that the time and space averaged properties of the medium are indeed anisotropic. One can therefore expect that the dynamic properties of turbulence differ between the horizontal and vertical directions. As the excitation of radial $p$ modes is predominantly governed by turbulent elements moving in the vertical direction, an open question is whether one should consider $\chi_{k}^{z}$ rather than $\chi_{k}$ in Eqs. (3) and (4) when taking into account the dynamic properties of the turbulence. In the present work, we therefore characterise both $\chi_{k}^{z}$ and $\chi_{k}$ from a $3 \mathrm{D}$ simulation and assess the consequences of using either $\chi_{k}^{z}$ or $\chi_{k}$ in the calculation of $P$.

\subsection{A Gaussian function for $\chi_{k}$}

Stein (1967) and Musielak et al. (1994) suggested several analytical forms for $\chi_{k}(\omega)$. The Gaussian Function (GF hereafter) is the simplest choice and is defined as

$\chi_{k}(\omega)=\frac{1}{\omega_{k} \sqrt{\pi}} \mathrm{e}^{-\left(\omega / \omega_{k}\right)^{2}}$

where $\omega_{k}$ is its linewidth.

In the time domain, the Gaussian function, Eq. (8), is the Fourier transform of a Gaussian function whose linewidth is equal to $2 \tau_{k}$, where $\tau_{k}$ is a characteristic time correlation length. Hence $\omega_{k}$ and $\tau_{k}$ are related to each other as

$\omega_{k}=\frac{2}{\tau_{k}}$.

The characteristic time $\tau_{k}$ is usually associated with the characteristic correlation time-scale of an eddy with wavenumber $k$. As in Balmforth (1992), we define it as

$\tau_{k} \equiv \frac{\lambda}{k u_{k}}$

where the velocity $u_{k}$ of the eddy with wave number $k$ is related to the kinetic energy spectrum $E(k)$ by (Stein 1967)

$u_{k}^{2}=\int_{k}^{2 k} \mathrm{~d} k E(k)$.

The parameter $\lambda$ in Eq. (10) accounts for our lack of precise knowledge of the time correlation $\tau_{k}$ under stellar conditions.

In the calculation of $P$, a GF is usually assumed for $\chi_{k}$ (e.g. Goldreich \& Keeley 1997, Balmforth 1992). This assumption is equivalent to supposing that two distant points in the turbulent medium are uncorrelated.

In Sect. 3, we use a 3D simulation of the upper part of the solar convective zone to derive the $v$-dependencies of $\chi_{k}^{z}$ and $\chi_{k}$. Inferred $v$-dependencies of $\chi_{k}^{z}$ and $\chi_{k}$ are compared to that of the GF. We next determine several analytical forms for $\chi_{k}^{z}$ and $\chi_{k}$ that can better represent their $v$-dependencies.

\section{Constraints from the 3D simulation}

The analysis of a 3D simulation of the upper part of the solar convective zone provides constraints for several physical parameters that enter the theoretical expression for the energy supply rate $P$ injected into the solar $p$ modes (Eq. (1)). The constraints may be considered to be of two types:

- Static constraints (static refers to spatial and time averages) determine the actual wavenumber dependency of $E(k, z)$, the kinetic turbulent spectrum, and $E_{s}(k, z)$, the turbulent spectrum associated with the entropy. The static constraints also determine the depth profile of the wavenumber $k_{0}^{E}$ at which convective energy is injected into the turbulent inertial range of $E$ (as in Samadi et al. 2003 we assume that the wavenumber $k_{0}^{E_{s}}$, at which convective energy is injected into the turbulent inertial range of $E_{s}$, is equal to $k_{0}^{E}$ ). They also provide the depth dependence of $u^{2}$, the mean square velocity, $w^{2}$, the mean square vertical component of the velocity, $\tilde{s}^{2}$, the mean square values of entropy fluctuations and $\Phi=u^{2} / w^{2}$, the mean values of the anisotropy studied in Samadi et al. (2003).

- The dynamic constraints, on the other hand, concern the frequency component $\chi_{k}$ and $\chi_{k}^{z}$ (see Sect. 2.2).

The static constraints have been established in Samadi et al. (2003). Here we investigate the dynamic constraints.

\subsection{The 3D simulation}

We study a 3D simulation of the upper part of the solar convection zone obtained with the 3D numerical code developed by Stein \& Nordlund (1998).

The simulated domain is $3.2 \mathrm{Mm}$ deep and its surface is $6 \times 6 \mathrm{Mm}^{2}$. The grid of mesh points is $256 \times 256 \times 163$ (i.e. $\sim 23 \mathrm{~km} \times 23 \mathrm{~km} \times 37 \mathrm{~km}$ ), the total duration $27 \mathrm{mn}$ and the sampling time 30 s.

Outputs of the simulation considered in Samadi et al. (2003) are the velocity field $\boldsymbol{u}(x, y, z, t)$ and the entropy $s(x, y, z, t)$ where - as in Samadi et al. (2003) $-z=r-R_{\odot}$ and $R_{\odot}$ is the radius at the photosphere (i.e. where $T=T_{\text {eff }}$ ). The quantities $\boldsymbol{u}(x, y, z, t)$ and $s(x, y, z, t)$ were used to determine the quantities $E(k, z), E_{s}(k, z), w$ and $\tilde{s}^{2}$ involved in the theoretical expression for the excitation rate $P$. In the present work we use the velocity field $\boldsymbol{u}(x, y, z, t)$ to characterise $\chi_{k}$ and $\chi_{k}^{z}$.

\subsection{Fourier transform}

At five different layers of the simulated domain, we compute the 3D Fourier transform, with respect to time and in the horizontal plane, of the velocity field $\boldsymbol{u}$. These layers cover a region where modes with frequency $v \gtrsim 2 \mathrm{mHz}$ are predominantly excited.

This provides $\hat{\boldsymbol{u}}(\boldsymbol{k}, z, v)$ where $\boldsymbol{k}$ is the wavenumber in the horizontal plane. Next we integrate $\hat{\boldsymbol{u}}^{2}(\boldsymbol{k}, z, v)$ over circles with radius $k=\|\boldsymbol{k}\|$ at each given layer $z$. This yields $\hat{\boldsymbol{u}}(k, z, v)$ and therefore $E(k, v, z) \equiv \hat{\boldsymbol{u}}^{2}(k, v, z)$. The quantity $\chi_{k}(v, z)$ is the 
frequency component of $E(k, v, z)$, at fixed $k$. Hence, according to Eqs. (6) and (7), $\chi_{k}(v, z)$ is obtained from $E(k, v, z)$ as:

$\chi_{k}(v, z)=\frac{E(k, v, z)}{\int \mathrm{d} v E(k, v, z)}$

where the integration over $v$ is performed over the frequency range $\left[-v_{\max }, v_{\max }\right]$ corresponding to the window of the Fourier analysis with respect to time $\left(v_{\max } \simeq 16 \mathrm{mHz}\right)$.

We proceed in the same manner for the vertical component of $\boldsymbol{u}$. This then provides $E_{z}(k, v, z) \equiv \hat{u}_{z}^{2}(k, v, z)$ and $\chi_{k}^{z}(v, z)$.

\subsection{Inferred properties of $\chi_{k}$ and $\chi_{k}^{z}$}

Figure 1 presents $\chi_{k}(v)$ as it is obtained from the simulation for the wavenumber $k$ at which $E(k, z)$ peaks $\left(k=k_{0}\right)$.

At the top of the superadiabatic region (for instance $z=$ $-0.4 \mathrm{Mm}$ in Fig. 1, this is the layer where the excitation is the largest), the GF does not correctly model $\chi_{k}(v)$ (see Fig. 1). However the discrepancies between the GF and the simulation data occur mostly above the solar cut-off frequency $(v \sim 5.5 \mathrm{mHz})$. Discrepancies between the GF and the 3D simulation data have then minor consequences for the $p$ modes excitation in this region. This is not the case deeper in the simulation where the largest discrepancies between the GF and the simulation data occur in the frequency range where the dominant amount of acoustic energy is injected into the $p$ modes $(v \sim 2-4 \mathrm{mHz})$.

To reproduce the shape of $\chi_{k}(v)$ obtained with the 3D simulation, one needs a function which at high frequency decreases more slowly than the GF. For modeling the $v$-dependency of $\chi_{k}(v)$, we thus propose three analytical functions: the Lorentzian function (LF hereafter)

$\chi_{k}(\omega)=\frac{1}{\pi \omega_{k} / 2} \frac{1}{1+\left(2 \omega / \omega_{k}\right)^{2}}$,

the Gaussian plus an Exponential Function (GEF hereafter)

$\chi_{k}(\omega)=\frac{1}{2}\left(\frac{1}{\omega_{k} \sqrt{\pi}} \mathrm{e}^{-\left(\omega / \omega_{k}\right)^{2}}+\frac{1}{2 \omega_{k}} \mathrm{e}^{-\left|\omega / \omega_{k}\right|}\right)$,

and the Gaussian plus a Lorentzian function (GLF hereafter)

$\chi_{k}(\omega)=\frac{1}{2}\left(\frac{1}{\omega_{k} \sqrt{\pi}} \mathrm{e}^{-\left(\omega / \omega_{k}\right)^{2}}+\frac{1}{\pi \omega_{k}} \frac{1}{1+\left(\omega / \omega_{k}\right)^{2}}\right)$.

All these functions satisfy the condition of normalisation of Eq. (7).

We first assume a constant $\lambda=1$. As shown in Fig. 1, all these non-Gaussian functions reproduce the $v$-variation of $\chi_{k}$ better than that obtained using a GF.

In the middle of the excitation region $(-0.5 \mathrm{Mm} \lesssim z \lesssim$ $0.0 \mathrm{Mm}$ ) the overall best agreement is obtained with the LF. Below $z \sim-0.5 \mathrm{Mm}$, the LF does not reproduce $\chi_{k}$ well enough but still reproduces its $v$-variation better than the other models.

However we have so far assumed that $\lambda$ (or equivalently the eddy time correlation) is depth independent, which is a strong assumption. When $\lambda$ is allowed to vary with $z$, we find that decreasing the value of $\lambda$ below $z \lessgtr-0.5 \mathrm{Mm}$, the LF best models $\chi_{k}$ below $z \sim-0.5 \mathrm{Mm}$ (e.g. $\lambda=1.6$ at $z=-0.64 \mathrm{Mm}$ and $\lambda \simeq 1.30$ at $z=-0.99 \mathrm{Mm}$, see Fig. 2). This shows that the variation with depth of the characteristic time $\tau_{k}$ (or equivalently the characteristic frequency $\omega_{k}$ ) is not correctly represented by the relations of Eqs. (10) and (11) when computed assuming a constant $\lambda$ below $z \sim-0.5 \mathrm{Mm} ; \tau_{k}$ increases faster with depth than expected from the relations (10) and (11). It is however found in Sect. 4 that this feature has negligible effect on $P$.

The function $\chi_{k}^{z}$ also decreases with the frequency more slowly than the GF (see Fig. 1). Moreover, decreasing values of $\lambda$ for $z \lesssim-0.5 \mathrm{Mm}$ provide a better fit of $\chi_{k}^{z}$. But in contrast with $\chi_{k}, \chi_{k}^{z}$ is overall better modeled with the GEF for $z \gtrsim-0.5 \mathrm{Mm}$ and with the GLF for $z \lesssim-0.5 \mathrm{Mm}$ rather than with the LF (not shown).

We conclude from the frequency analysis of the 3D simulation that the simple Gaussian function cannot correctly represent the actual dynamic properties of the turbulent medium. One may expect that the GF causes an underestimation of the acoustic energy injected into the solar $p$ modes. Instead the frequency analysis favours a non-Gaussian function for $\chi_{k}^{z}$ and $\chi_{k}$ that decreases more slowly with $v$ than the GF.

\section{Consequences in terms of $p$ modes excitation}

\subsection{Computations of the excitation rate $P$}

Computation of the excitation rate $P$ is performed as in Samadi et al. (2003) except that here two analytical functions other than the GF are assumed for $\chi_{k}$, as discussed in Sect. 3. The computation process is summarised as follows: The eigenfunctions $\left(\xi_{r}\right)$ and their frequencies $(v)$ are computed with Balmforth's (1992) non-adiabatic code for a solar 1D mixing-length model based on Gough's (1977) non-local timedependent formulation of convection.

The quantities $\Phi, w^{2}$ and $s^{2}$ are obtained from the 3D simulation. The $k$-dependency of $E(k, z)$ is the Extended Kolmogorov Spectrum (EKS hereafter) defined as:

$E(k) \propto\left(k / k_{0}\right)^{+1}$ for $k_{0}>k>k_{\min }$
$E(k) \propto\left(k / k_{0}\right)^{-5 / 3}$ for $\quad k>k_{0}$.

In Eq. (16), the wavenumber $k_{0}$ is the wavenumber at which $E(k)$ peaks and $k_{\min }$ is the minimal wavenumber reached by the $3 \mathrm{D}$ simulation $\left(k_{\min }=1.05 \mathrm{Mm}^{-1}\right)$. The variation with depth of $k_{0}$ is also given by the $3 \mathrm{D}$ simulation. The $k$ dependency of the EKS reproduces the global features of $E$ arising from the $3 \mathrm{D}$ simulation. The same model is considered for $E_{s}(k, z) . E(k, z)$ and $E_{s}(k, z)$ satisfy the normalisation conditions:

$$
\begin{aligned}
& \int_{k_{\min }}^{+\infty} \mathrm{d} k E(k, z)=1 / 2 \Phi w^{2} \\
& \int_{k_{\min }}^{+\infty} \mathrm{d} k E_{s}(k, z)=1 / 2 \tilde{s}^{2}
\end{aligned}
$$

The total energy contained in $E(k, z)$ and $E_{s}(k, z)$, and their depth dependencies, are then obtained from the $3 \mathrm{D}$ simulation according to Eq. (17). These theoretical estimates for $P$ are then 

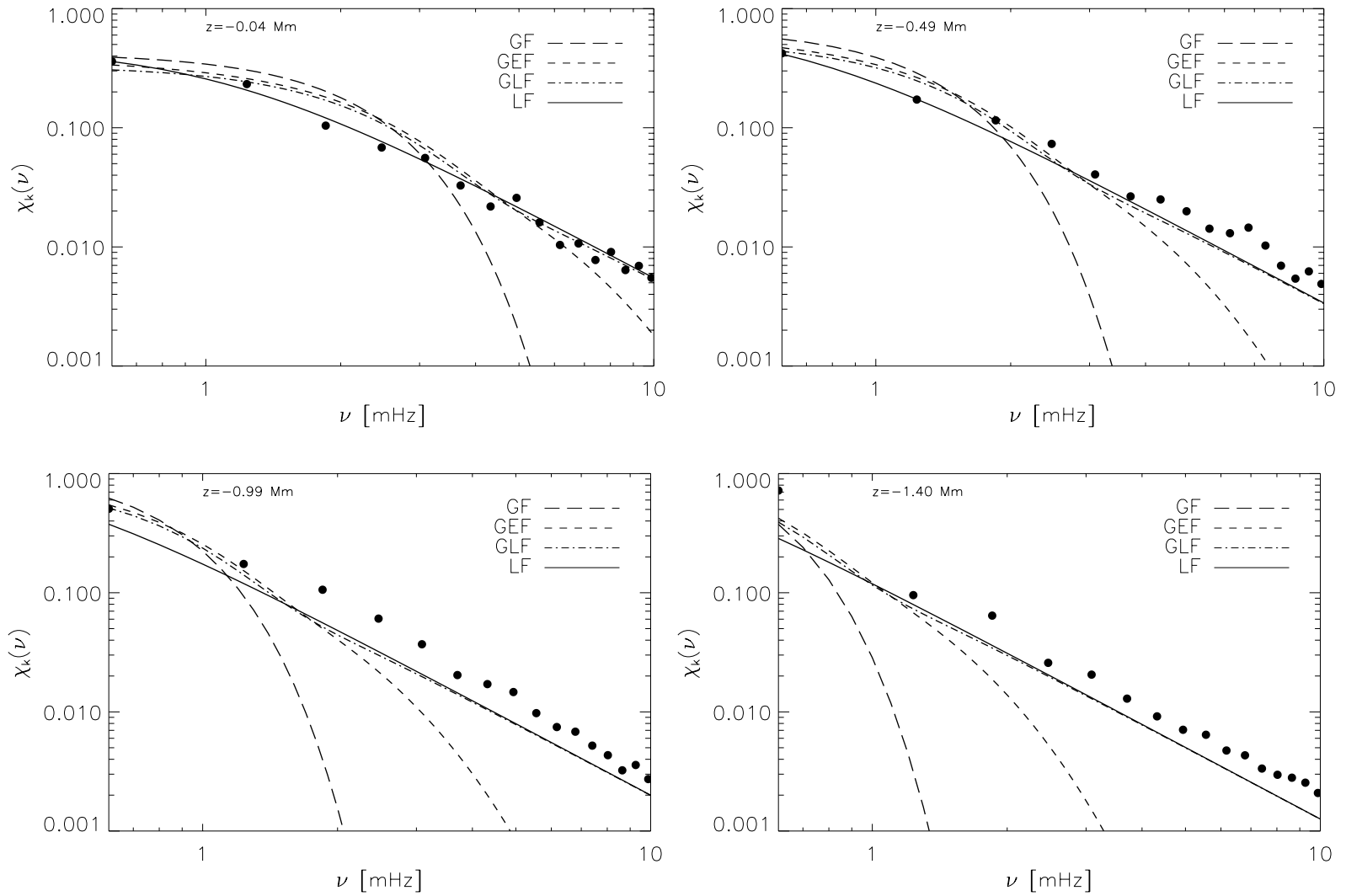

Fig. 1. The filled dots represent $\chi_{k}(v)$ obtained from the simulation for the wavenumber $k_{0}$ at which $E(k, z)$ peaks. The solid curves represent the Lorentzian function (LF, Eq. (13)), the dots-dashed curves the Gaussian Lorentzian function (GLF, Eq. (15)), the dashed curves the Gaussian Exponential function (GEF, Eq. (14)) and the long dashed curves the Gaussian function (GF, Eq. (8)). In these four analytical functions, $\lambda=1$ is assumed for the calculation of $\omega_{k}$ (Eqs. (10) and (11)). Four different layers are considered: $z=-0.04 \mathrm{Mm}$ (the top of the superadiabatic region), $z=-0.49 \mathrm{Mm}, z=-0.99 \mathrm{Mm}$ and $z=-1.40 \mathrm{Mm}$.

compared with the "observed" $P$ from Chaplin et al. (1998)'s seismic data, calculated according to the relation:

$P\left(\omega_{0}\right)=2 \eta \frac{I}{\xi_{r}^{2}\left(r_{s}\right)} v_{s}^{2}\left(\omega_{0}\right)$

where $r_{s}$ is the radius at which oscillations are measured,

$I \equiv \int_{0}^{M} \mathrm{~d} m \xi_{r}^{2}$

is the mode inertia and where the mode damping rate $(\eta)$ and the mode surface velocity $\left(v_{s}\right)$ are obtained from Chaplin et al. (1998). In Eq. (18), the mode mass $I / \xi_{r}^{2}\left(r_{s}\right)$ is given by the GMLT model and we adopt $r_{s}=R_{\odot}+200 \mathrm{~km}$ consistently with Chaplin et al. (1998)'s observations.

\subsection{Comparisons with observations}

We investigate the effect of using different analytical functions for $\chi_{k}$ (Sect. 3 ) in the computation of $P$. We first assume a constant value $\lambda=1$. Results are shown in Fig. 3. Computations performed with the GF underestimate the observed $P$ values by a factor $\sim 2.7$. On the other hand, the LF, GEF and GLF choices result in larger values for the computed $P$ than the GF one ( $\sim 2$ times larger). This brings them closer to the observations, compared with the GF choice for $\chi_{k}$. The reason is that all the non-Gaussian functions (the LF, the GEF and the GLF) - which indeed better model $\chi_{k}^{z}$ and $\chi_{k}$ from the 3D simulation than does the GF - decrease more slowly with $v$ than the GF in the frequency range where the mode amplitudes are large $(v \simeq 2-4 \mathrm{mHz}$ ). Consequently a larger amount of acoustic energy is injected into the modes with the non-Gaussian functions than with the GF.

In Sect. 3.3, the overall best models for $\chi_{k}$ were obtained with the LF and with decreasing values of $\lambda$ below $z \sim-0.5 \mathrm{Mm}$. We use a simple model for the depth variation of $\lambda$ :

$$
\begin{array}{ll}
\lambda=1 & \text { for } z>-0.5 \mathrm{Mm} \\
\lambda=0.9+0.71(0.64 \mathrm{Mm}+z) & \text { for }-0.5 \geq z \geq-1 \mathrm{Mm} \\
\lambda=0.35 & \text { for } z<-1 \mathrm{Mm}
\end{array}
$$

We have computed $P$ according to the simple model of Eq. (20) and assuming the LF. We find no significant changes for $P$ compared to the calculations in which a constant value $\lambda=1$ is assumed (not shown).

In Sect. 3 we found that $\chi_{k}^{z}$ is better modelled with the GEF for $z \gtrsim-0.5 \mathrm{Mm}$ and with the GLF for $z \lesssim-0.5 \mathrm{Mm}$ rather than with the LF. However, as the stochastic excitation is the largest in the range $-0.5 \mathrm{Mm} \lesssim z \lesssim 0 \mathrm{Mm}$, we can assume the GEF in all the domain. The LF results in a value for $P_{\max }$ slightly larger than the one resulting from the GEF 

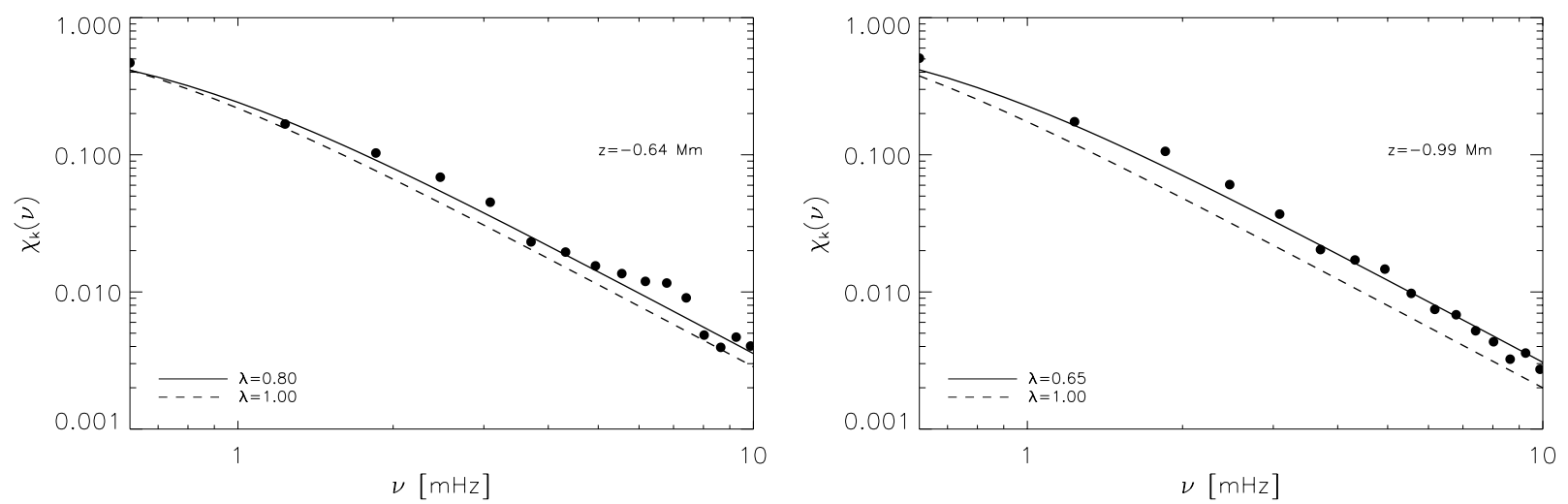

Fig. 2. As in Fig. 1, the filled dots represent $\chi_{k}(v, z)$ obtained from the simulation at two different layers: $z=-0.64 \mathrm{Mm}$ (left panel) and $z=-0.99 \mathrm{Mm}$ (right panel). The other curves represent the LF (Eq. (15)) with different assumptions for $\lambda$ : The dashed curved correspond to $\lambda=1$ and the solid curves correspond to $\lambda=0.80$ at $z=-0.64 \mathrm{Mm}$ (left panel) and $\lambda=0.65$ at $z=-0.99 \mathrm{Mm}$ (right panel).

(only $\sim 1.2$ larger). A better agreement is then obtained with the analytical functions which fits best $\chi_{k}$ (i.e. the LF) than the one which fits best $\chi_{k}^{z}$ (i.e. the GEF) in contrast with the intuitive idea mentioned in Sect. 2.2 that the excitation of radial $p$ modes depends rather on the properties of $\chi_{k}^{z}$ than on those of $\chi_{k}$.

In the frequency range where observational constraints are available, differences between results obtained with the different adopted non-Gaussian functions are of the same order as the actual error bars associated with the observed $P$ values.

Below the frequency range of the observations - i.e. below $v \lesssim 1.8 \mathrm{mHz}$ - the differences between the different nonGaussian functions are very large compared to the error bars (see bottom panel of Fig. 3). Those differences are related to differences in the $v$-variation of the non-Gaussian models. Observational constraints at low frequency could therefore confirm that the LF is indeed the best representation for $\chi_{k}(v)$.

Important discrepancies still remain at high frequency $(v \gtrsim$ $3.5 \mathrm{mHz}$ ). The excitation rate derived from the observations decreases as $\sim v^{-6.2}$ above $v \simeq 3.5 \mathrm{mHz}$ ) whereas the computed $P$ decreases as $\sim v^{-1}$ (see bottom panel of Fig. 3). Possible origins of this discrepancy are discussed in Sect. 5.5.

Another consequence of a non-Gaussian dependence of $\chi_{k}\left(\right.$ or $\chi_{k}^{z}$ ) with the frequency is a larger relative contribution of the Reynolds stress $P_{R}$ to the mode excitation rate $P$. This is shown in Fig. 4. The GF generates a relative contribution of the Reynolds stress to the excitation $\left(P_{R} / P\right)$ which is smaller than that obtained assuming a non-Gaussian function (e.g. for the LF, the relative contribution of $P_{R}$ to the excitation is at least $\sim 2$ times larger than with the GF). Excitation by the entropy fluctuations takes place predominantly at the top of the excitation region over a thin layer $(\lessgtr 0.2 \mathrm{Mm})$ while that due to the Reynolds stress extends deeper below $(\sim 0.5-2 \mathrm{Mm})$. At the top of the excitation region, the discrepancy between the GF and $\chi_{k}$ inferred from the 3D simulation mainly occurs above $v \simeq 5 \mathrm{mHz}$ and thus has a small impact on mode excitation. This is not the case deeper in the excitation region where the GF under-estimates $\chi_{k}$ in a frequency range increasing inward.

\section{Conclusion and discussion}

\subsection{A Non-Gaussian eddy time correlation}

In the present work we characterize empirically $\chi_{k}$ and of $\chi_{k}^{z}$, the frequency components of the correlation product of the turbulent velocity field and of its vertical component respectively. A frequency analysis of a solar 3D simulation shows that at large scales $\left(k \sim k_{0}\right)$ the Gaussian function significantly underestimates $\chi_{k}$ and $\chi_{k}^{z}$ in the frequency range $(v \simeq 2-4 \mathrm{mHz})$ where acoustic energy injected into the solar $p$ modes is the largest.

As a result, the maximum value of $P$ is found $\sim 2.7$ smaller than the solar seismic constraints.

This partly explains the underestimate of the values of solar $p$ mode excitation rates obtained by Houdek et al. (1999) whose computations are based on the theoretical expression by Balmforth (1992) and the underestimate obtained by Samadi et al. (2003).

In order to reproduce the main properties of $\chi_{k}$ (or $\chi_{k}^{z}$ ), one has to consider a model which must decrease more slowly with $v$ than the GF. We then assume for $\chi_{k}$ and $\chi_{k}^{z}$ three different simple analytical forms: the Lorentzian Function (LF), the socalled "Gaussian Exponential Function" (GEF, which is composed by the GF plus an exponential function) and the so-called "Gaussian Lorentzian Function" (GLF, which is composed by the GF plus a Lorentzian function).

From the top of the excitation region (which corresponds to the top of the superadiabatic region) down to the middle of the excitation region $(z \sim-0.5 \mathrm{Mm}$ where $z$ is the distance to the radius at the photosphere), the best agreement between $\chi_{k}$ and the analytical approximations is obtained with the LF and with $\lambda=1$. Deeper within the excitation region $(z \lesssim-0.5 \mathrm{Mm})$, the agreement is better for $\lambda<1$ and $\lambda$ decreasing with depth.

The frequency dependencies of $\chi_{k}^{z}$ and $\chi_{k}$ are found to be very similar. However $\chi_{k}^{z}$ is best modeled by the GEF. As for $\chi_{k}$, the agreement is better below $z \sim-0.5 \mathrm{Mm}$ with decreasing values of the parameter $\lambda$ than with $\lambda=1$.

Assuming a non-Gaussian function - either the LF, the GEF or the GLF - results in values for $P_{\max }$, the maximum of excitation power, which are $\sim 2$ times larger than when assuming the 

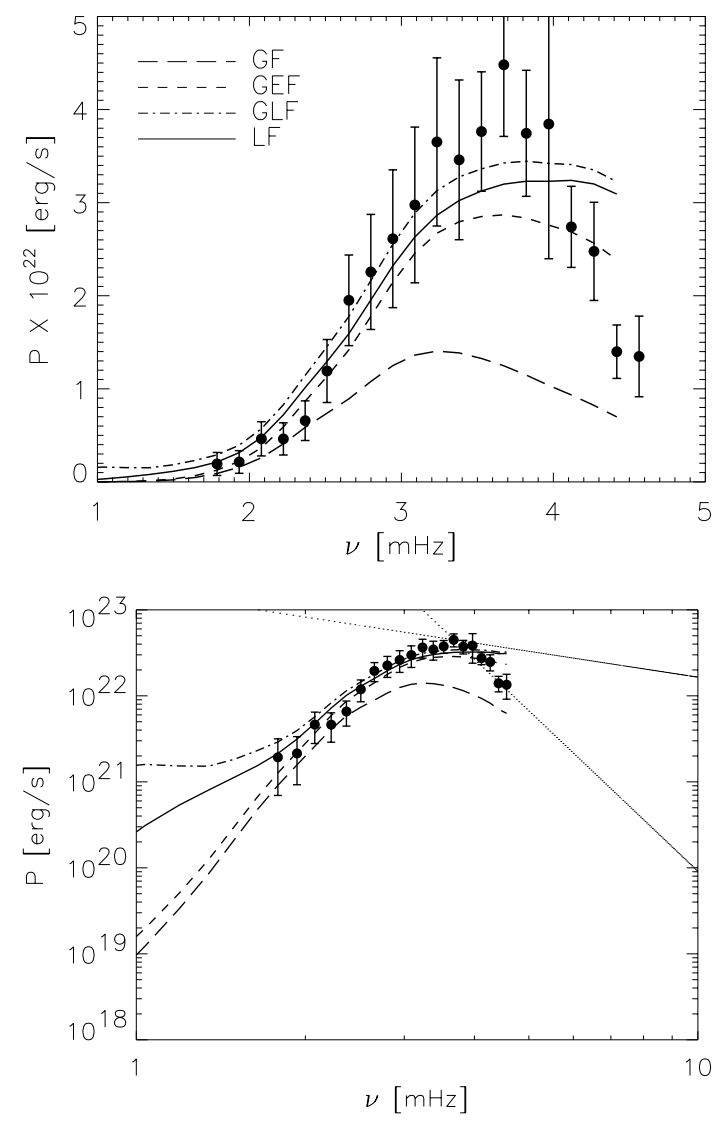

Fig. 3. Top: The curves correspond to computed values of $P(v)$ obtained with different analytical functions for $\chi_{k}(v)$ : the GF (long dashed curve), the GEF (dashed curve), the GLF (dots-dashed curve) and the LF (solid curve). In all calculations, we assume $\lambda=1$. The dots represent $P(v)$ derived from the amplitudes and line widths of the $\ell=0 p$ modes measured by Chaplin et al. (1998). Bottom: same as the top panel but $P$ is plotted in a log-log representation as it is usually represented in the literature. The vertical and horizontal scales have been chosen for an easy comparison with equivalent plots found in Stein \& Nordlund (2001). The lines with dots show two different power laws $v^{p}$ : one with $p=-6.2$ and the other with $p=-1$.

GF and brings $P_{\max }$ much closer to the maximum of $P$ derived from the solar seismic data of Chaplin et al. (1998).

We also find that taking into account the variation of $\lambda$ with depth for $z$ below $-0.5 \mathrm{Mm}$ does not significantly change the values of $P$. A constant value can then be assumed in the calculation of the solar $p$ mode excitation rates. The constant value of $\lambda$ on the other hand plays an important role and we find $\lambda=1$.

We have investigated the sensitivity to the adopted representation for $\chi_{k}$ : Although the LF fits best the $v$-variation of $\chi_{k}$ inferred from the 3D simulation, the GLF results in value for $P_{\max }$ closer to the seismic constraints. However, the differences obtained with the different non-Gaussian approximations for $\chi_{k}$ are globally smaller than the actual error bars associated with the observations of Chaplin et al. (1998). On the other hand, below the frequency range where observational constraints on $P$ are available (i.e. below $v \lesssim 1.8 \mathrm{mHz}$ ), the differences between $P$ obtained with different non-Gaussian functions are very large compared to the current error bars. Those

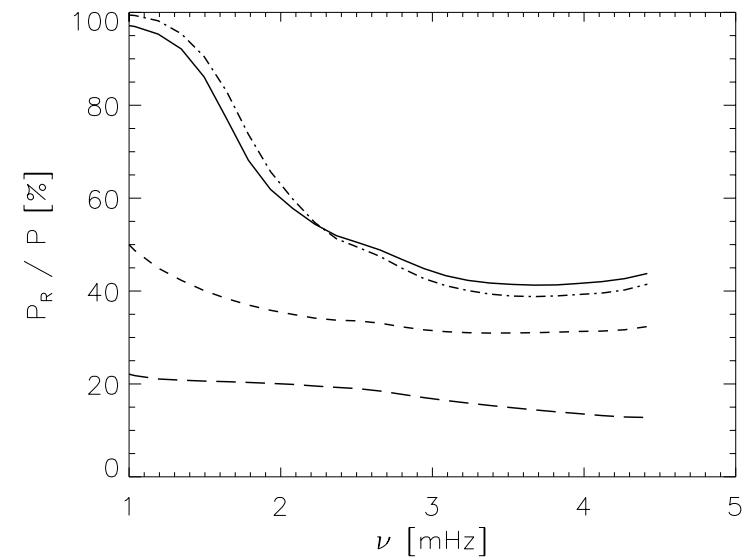

Fig. 4. Top: Same as Fig. 3 for the relative contribution of the Reynolds stress, $P_{R}$ to the total acoustic energy $P$.

differences are directly related to the diffences in the $v$-variation of the non-Gaussian forms investigated in this work. This suggests that accurate enough data below this frequency range, could provide confirmation that the LF is indeed the best model for $\chi_{k}$.

\subsection{Relative contribution of the entropy fluctuations to the excitation}

The non-Gaussian character of $\chi_{k}$ causes the excitation region to extend deeper $(\sim 500 \mathrm{~km}$ for modes of order $n=20)$ than with the GF ( $\sim 200 \mathrm{~km}$ resp.). The largest entropy fluctuations mainly occur at the outermost part of the convective zone (CZ) over a very thin region $(\sim 100 \mathrm{~km})$ while excitation by the Reynolds stress contribution occurs on a more extended region. Consequently the non-Gaussian property of $\chi_{k}$ leads to a relatively larger contribution of the Reynolds stress to the excitation than in the case of a GF. As a result, the Reynolds stress contribution is of the same order as the contribution arising from the advection of the turbulent fluctuations of entropy by the turbulent movements (the so-called entropy source term). This is in contrast with previous results (Samadi et al. 2001) based on the GF which concluded that the entropy source term dominates the Reynolds stress by about $\sim 20$. It also differs with results by Goldreich et al. (1994) who found that the excitation arising directly from the entropy fluctuations dominates by about $\sim 10$.

On the other hand, in Stein \& Nordlund (2001), the excitation by turbulent pressure (Reynolds stress) is found dominant ( $\sim 4$ times larger) whereas here we find that the contribution of the entropy source term cannot be neglected. Whether this is the signature of some deficiency in the present excitation model is an open question.

\subsection{Summary}

We show that the usually adopted Gaussian function for $\chi_{k}$ is neither consistent with the properties of $\chi_{k}$ inferred from the $3 \mathrm{D}$ simulation nor does it reproduce the observed maximum of the solar $p$-modes excitation rates. 
Following an empirical approach we improve the model of the convective eddy time-correlation $\chi_{k}$ which enters the current model of stochastic excitation. We then show that to reproduce both the $v$-variation of $\chi_{k}$ as inferred from the 3D simulation and the observed maximum of the solar $p$-modes excitation rates one has to consider a non-Gaussian form which decreases at high frequency slower than the GF, as do the different non-Gaussian functions investigated here.

The use of non-Gaussian functions, for instance the LF, reproduces reasonably well the maximum value of the rate at which solar $p$-modes are excited without any adjustments of free parameters or without introducing a scaling factor, in contrast with previous approaches (e.g. Balmforth 1992; Goldreich et al. 1994; Samadi et al. 2001). We then solve the problem of the underestimation by the previous theoretical approaches. Furthermore the use of such a non-Gaussian form for $\chi_{k}$ makes the contribution of the turbulent pressure to the excitation much larger than in previous works making our results more consistent with that by Stein \& Nordlund (2001).

Our investigation clearly emphasizes the non-Gaussian character of the solar p modes excitation as a result of the nonGaussian property of the convective eddies time-correlations. It also shows that the dynamic properties of the solar turbulent convection inferred from the 3D simulation are consistent with the helioseismic data.

We stress that only simple non-Gaussian forms for $\chi_{k}$ have been investigated here. More sophisticated forms are likely to improve the agreement with the $v$-dependency of $\chi_{k}$ (or $\chi_{k}^{z}$ ). This would not affect the main conclusions presented in the present paper.

\subsection{Possible origin of the non-Gaussian property of $\chi_{k}$}

We recall that $\chi_{k}$ measures the temporal evolution of the correlation between two points of the turbulent medium separated by a distance of $\sim 2 \pi / k$. A Gaussian time-correlation means that the fluid motions in the medium are random in time. Departure from a Gaussian time-correlation at large scales $\left(k \sim k_{0}\right)$ suggests that a strong correlation exists at that scale.

Downward plumes are likely to be responsible for the nonGaussian behaviour of $\chi_{k}$. Downward and upward convective motions are indeed highly asymmetric (Stein \& Nordlund 1998): downward flows are associated with patterns (plumes) which are more coherent than the upward moving structures (Rieutord \& Zahn 1995). The upward flows are associated with less coherent and more random structures (granules) characterised by a broad variety of sizes and lifetimes (Rieutord $\&$ Zahn 1995). The non-Gaussian behavior of $\chi_{k}$ can most probably be attributed to plumes. This however remains to be checked (work in progress).

\subsection{Possible origin of the remaining discrepancy}

Despite a clear improvement in the agreement between observed and theoretical excitation rates, important discrepancies between the computed $P$ and the solar measurements still remain at high frequency $v \gtrsim 3.5 \mathrm{mHz}$ (see Sect. 4.2 and Fig. 3).
On the "observational side", at high frequency, larger uncertainties for the damping rates $\eta$ induce larger uncertainties on the derived supply energy rates.

On the theoretical side, part of the discrepancy might well be attributed to a poor description of the eigenfunctions at high frequency. Indeed, the discrepancies between the calculated eigenfrequencies and the observed ones are largest at high frequency $(v \gtrsim 3 \mathrm{mHz})$. This indicates that the description of the eigenfunctions are less accurate at high frequency. As the expression for calculating $P$ involves the first and second derivatives of the mode eigenfunction, the lack of accuracy in the calculation of the eigenfunctions has a larger impact on $P$ at high frequencies than at small frequencies.

Other possible causes can perhaps be related to our simplified excitation model which assumes isotropic turbulence. Indeed the current theory assumes that the stochastic excitation is the same in all three directions, particularly between the ascending and descending flows. However the kinetic energy and entropy fluctuations are larger in the downward flows than in the upward flows (Stein \& Nordlund 1998). Therefore the driving arising from the advection of the turbulent fluctuations of entropy by the turbulent movements differs significantly between the elements moving downwards and those moving upwards. As the entropy fluctuations are largest in the outermost part of the convective zone, the above mentioned asymmetry will predominantly affect the high frequency modes.

Moreover, it is also assumed that the total kinetic energy, $E$, is isotropically injected in all 3 directions. Excitation of the radial $p$ modes results from the vertical component of the velocity. However at the top of the convective zone, the distribution of kinetic energy in $E(k, z)$ and in $E_{z}(k, z)$ are very different from each other. These differences may affect more strongly the high frequency modes. Consequences of these departures from the isotropic assumption need to be further investigated.

\subsection{Perspectives}

The non-Gaussian property of $\chi_{k}$ and its consequences for the stochastic excitation has been investigated so far only for the Sun. However such a non-Gaussian feature of the turbulence will most likely also be of importance for solar-like oscillating stars more massive than the Sun, provided our analysis is also valid for these stars. This can substantially change the excitation spectrum $P$ for such stars compared to that which is currently predicted.

Therefore investigations of $p$ mode excitation in hotter and more massive stars must be undertaken, which should proceed in two steps: first, the validity of the present results obtained in the solar case must be investigated for other stars with, for instance, the help of dedicated 3D simulations. The conclusions which will drawn from this first step must be used in a second step to study the frequency dependence and the magnitude of $P$ for different solar-like oscillating stars (see preliminary results in Samadi et al. 2002).

Future space missions such as COROT (Baglin \& The Corot Team 1998), MOST (Matthews 1998) and Eddington (Favata et al. 2000) will provide high-quality data on seismic 
observations. COROT will be the first mission that will provide high precision mode amplitudes and linewidths in other stars. This high-quality data will allow us to derive the excitation rate $P$ and will provide improved observational constraints on the theory of stochastic excitation which is, at present, poorly constrained by observation.

Acknowledgements. We thank H.-G. Ludwig for useful discussions and valuable help in analysing the simulated data and we are indebted to G. Houdek for providing us the 1D solar model. We gratefully thank our referee (T. Appourchaux) for pertinent remarks which led to an improvement of the paper. RS's work has been supported in part by the Particle Physics and Astronomy Research Council of the UK under grant PPA/G/O/1998/00576.

\section{References}

Baglin, A., \& The Corot Team. 1998, in New Eyes to See Inside the Sun and Stars, IAU Symp., 185, 301

Balmforth, N. J. 1992, MNRAS, 255, 639

Batchelor, G. K. 1970, The theory of homogeneous turbulence (University Press)

Chaplin, W. J., Elsworth, Y., Isaak, G. R., et al. 1998, MNRAS, 298, L7

Favata, F., Roxburgh, I., \& Christensen-Dalsgaard, J. 2000, in The Third MONS Workshop: Science Preparation and Target Selection, 49
Goldreich, P., \& Keeley, D. A. 1977, ApJ, 212, 243

Goldreich, P., Murray, N., \& Kumar, P. 1994, ApJ, 424, 466

Gough, D. O. 1977, ApJ, 214, 196

Houdek, G., Balmforth, N. J., Christensen-Dalsgaard, J., \& Gough, D. O. 1999, A\&A, 351, 582

Matthews, J. M. 1998, in Structure and Dynamics of the Interior of the Sun and Sun-like Stars, 395

Musielak, Z. E., Rosner, R., Stein, R. F., \& Ulmschneider, P. 1994, ApJ, 423, 474

Osaki, Y. 1990, in Lecture Notes in Physics: Progress of Seismology of the Sun and Stars, ed. Y. Osaki, \& H. Shibahashi (SpringerVerlag), 75

Rieutord, M., \& Zahn, J.-P. 1995, A\&A, 296, 127

Samadi, R. 2001, in SF2A-2001: Semaine de l'Astrophysique Francaise, E148 [astro-ph/0108363]

Samadi, R., Nordlund, Å., Stein, R., Goupil, M.-J., \& Roxburgh, I. 2003, A\&A, 403, 303

Samadi, R., \& Goupil, M. 2001, A\&A, 370, 136 (Paper I)

Samadi, R., Goupil, M., \& Lebreton, Y. 2001, A\&A, 370, 147

Samadi, R., Nordlund, A., Stein, R. F., Goupil, M.-J., \& Roxburgh, I. 2002, in SF2A-2002: Semaine de l'Astrophysique Francaise, meeting held in Paris, France, June 24-29, ed. F. Combes, \& D. Barret (EDP Sciences)

Stein, R. F. 1967, Sol. Phys., 2, 385

Stein, R. F., \& Nordlund, A. 1998, ApJ, 499, 914

Stein, R. F., \& Nordlund, Å. 2001, ApJ, 546, 585

Tennekes, H., \& Lumley, J. 1982, A first course in turbulence, 8th edition (MIT Press) 